\title{
Efficacy of a Biorevitalizing-Filler Solution on All Skin Aspects: 10 Years Approach through in Vitro Studies and Clinical Trials
}

\author{
Sophie Robin1, Férial Fanian ${ }^{2 *}$, Carol Courderot-Masuyer1, Michel Tordjman ${ }^{3}$, \\ Frederic Braccini ${ }^{4}$, Sylvie Boisnic ${ }^{5}$, Valérie Philippon'2, Anne Grand Vincent ${ }^{3}$, \\ Catherine Salomon ${ }^{6}$, Michel Manfait ${ }^{7}$, Philippe Humbert ${ }^{8}$, Philippe Piccerelle ${ }^{9}$ \\ ${ }^{1}$ Bioexigence SAS, Besançon, France \\ ${ }^{2}$ Laboratoires Fill-Med, Paris, France \\ ${ }^{3}$ Aesthetic Doctor, Paris, France \\ ${ }^{4}$ Aesthetic Surgeon, Nice, France \\ ${ }^{5}$ Gredeco SARL, Paris, France \\ ${ }^{6}$ Laboratoires Multaler, Argenteuil, France \\ ${ }^{7}$ Reims University, Pharmaceutical Faculty, Reims, France \\ ${ }^{8}$ University of Bourgogne-Franche Comté, Besançon, France \\ ${ }^{9}$ IMBE, UMR CNRS 7263 IRD 237 University of Aix-Marseille, Pharmaceutical Faculty, Marseille, France \\ Email: ^fanian@gmail.com
}

How to cite this paper: Robin, S., Fanian, F., Courderot-Masuyer, C., Tordjman, M., Braccini, F., Boisnic, S., Philippon, V., Vincent, A.G., Salomon, C., Manfait, M., Humbert, P. and Piccerelle, P. (2021) Efficacy of a Biorevitalizing-Filler Solution on All Skin Aspects: 10 Years Approach through in Vitro Studies and Clinical Trials. Journal of Cosmetics, Dermatological Sciences and Applications, 11, 18-37.

https://doi.org/10.4236/jcdsa.2021.111003

Received: November 27, 2020

Accepted: March 5, 2021

Published: March 8, 2021

Copyright () 2021 by author(s) and Scientific Research Publishing Inc. This work is licensed under the Creative Commons Attribution International License (CC BY 4.0)

http://creativecommons.org/licenses/by/4.0/

cc) (i) Open Access

\begin{abstract}
Introduction: Skin aging is the result of many cellular dysfunctions over time particularly the fibroblasts and the keratinocytes. These dysfunctions could be decelerated by the preventive effects of some skin treatments such as Intradermal injections. NCTF $135 \mathrm{HA}^{\circledR}$ has a polycomponent formulation designed to improve the efficacy of non-cross linked hyaluronic acid (as a micro-filler) on fibroblasts function. Although NCTF 135HA has been used by aesthetic practitioners since 20 years, we have analyzed all in vitro, ex vivo and in vivo studies during past 10 years in order to summarized its anti-aging effect. Methods: In these series of studies, the known effects of HA have been evaluated with this boosted formula. Collagen I synthesis, antiglycation effect and contractile forces developed by fibroblasts were studied. Fibroblasts and keratinocytes proliferations were evaluated in monolayer cells culture. The filling effect thanks to NCTF 135HA injection was ex vivo performed by fringe projection and the protective effect of NCTF 135HA against solar irradiation was performed ex vivo to study proliferation rate, elastin and collagen expression. Two clinical trials were performed on women to evaluate the anti-aging effect of NCTF 135HA injection. Hydration, firmness, radiance, wrinkles size, pore size, thickness and density of the dermis were analyzed.
\end{abstract}


Results: Collagen I, anti-AGE products, cell proliferation and contractile forces were significantly increased with NCTF $135 \mathrm{HA}$ in culture medium. $E x$ vivo studies showed that NCTF 135HA increased, cells proliferation rate, elastin and collagen production. In addition, the filling effect of the NCTF $135 \mathrm{HA}$ is significantly superior to placebo. The first clinical trial shows that NCTF 135HA injection significantly increased tonicity, hydration and radiance of the skin and significantly decreased wrinkle score. The second clinical trial demonstrated that NCTF $135 \mathrm{HA}$ injection significantly increased dermis density and thickness, skin homogeneity and skin radiance and significantly decreased pore size and wrinkle depth and volume. Conclusion: In vitro, ex vivo and in vivo studies showed that this minimally invasive technique could be used in order to improve the skin quality which could have a visual effect on preventing the aging signs.

\section{Keywords}

Mesotherapy, Skin Rejuvenation, NCTF 135HA ${ }^{\circledR}$, Hyaluronic Acid, Skin Biorevitalization, Skin Booster

\section{Introduction}

Our knowledge of the skin as a complex, immune, multifunctional organ is constantly evolving, including our insights into the skin aging process [1]. Skin aging can be due to intrinsic and extrinsic processes, which contribute simultaneously to a progressive loss on skin integrity. Intrinsic factors were genetically determined and extrinsic factors were due to lifestyle and environment. Structural stability as well as physiological function is affected. Physiological changes in aged skin include structural and biochemical changes. The skin thins progressively over adult life at an accelerating rate [2]. The epidermis decreases in thickness particularly in women and particularly on the face, neck, chest, hands and forearms [3]. A reduction of the water content on the skin is also observed [4]. Dermis thickness decreases with age [1]. Skin aging can largely be attributed to dermal fibroblast dysfunction and a decrease in their number and biosynthetic activity. The amount of glycosaminoglycans on the dermis declines with age, as does the amount of hyaluronic acid produced by fibroblasts [5]. Aging is inevitably associated with a decrease in collagen turnover (due to a decrease in fibroblasts and their collagen synthesis) as well as elastin [6]. Reactive oxygen species (ROS) as a by-product of cellular metabolism due to oxidative stress generated by ultra-violet, tobacco, pollution exposure... ROS cause damage to critical cellular components like membranes, enzymes and DNA.

Aesthetic Medicine specialists (dermatologists, plastic surgeons, aesthetic doctors, etc.) are increasingly called to offer solutions that respond to the constant request for "eternal youth". Often not to use more heavy invasive surgical techniques such as dermabrasion, lifting surgery and laser resurfacing, today 
there is big tendency to use the injectable products to reduce or erase wrinkles and skin depressions, as well as to remodel certain parts of the body [7]. Intradermal multi-injection is an injection method based on multipuncture, to inject the treatment solution exactly where they have to be efficient, it means "the small quantities at the right place". It has more recently been used in aesthetic dermatology, where there has been an upturn in interest of this method due to the development of cutaneous anti-aging treatments [8].

Intradermal multi-injection is a technique that involves micro-injections of products such as hyaluronic acid, with or without vitamins, minerals and amino acids into the superficial layers of the skin [9]. This allows active and essential ingredients to come directly into contact with the dermal fibroblasts and keratinocytes that are key to the more favorable appearance of younger skin and have a beneficial effect on metabolic processes [10].

NCTF $135 \mathrm{HA}^{\circledR}$ is a CE-labelled class III medical device. Although it has been used since 1978 by the practitioners in France, it has been freely marketed in the European Union since September 2007 after obtaining his CE marking. This product is one of the pioneer products in this field and there are many similar products produced based on its formulation.

NCTF $135 \mathrm{HA}^{\circledR}$ is a hyaluronic acid (HA) based dermal filler indicated for revitalization and intense hydration of tired or dull skin, the filling of superficial wrinkles and the re-plumping of mature skin or skin that lacks firmness.

As it has been used widely by aesthetic practitioners as one of the most widely used polyrevitalizing solution since decades, we have decided to analyze all in vitro, ex vivo and in vivo studies on this product during past 10 years in order to verify its proved effects on different aspect of the skin.

\section{Product}

NCTF $135 \mathrm{HA}^{\circledR}$ contains two components:

- $15 \mathrm{mg}$ of non-cross-linked hyaluronic acid obtained through biotechnological processes. It is a linear polysaccharide identical to the HA present in connective tissue;

- Polyrevitalizing component, a biological solution developed to support the HA and its effects on the tissue. It is mainly composed of 5 groups of ingredients: vitamins, amino acids, mineral salts, coenzymes and nucleic acids.

This product is available in the form of the sterile and ready-to-use vials of 3 $\mathrm{ml}$, sterilized by double filtering method.

\section{In Vitro Studies}

\subsection{Effect of NCTF 135HA ${ }^{\circledR}$ on Collagen I Production}

\subsubsection{Objectives}

The objective of this study was to evaluate if the HA boosted by a polyrevitalizing component has still a positive effect on Collagen I production, the effect which has been already proved by HA alone [11]. 


\subsubsection{Materials and Methods}

The study performed by Bioalternatives Laboratory at Gençay, France. Normal human fibroblasts from 4 donors were seeded in 12-wells plate. After one day of culture at $37^{\circ} \mathrm{C}$ with $5 \%$ of $\mathrm{CO}_{2}$, two dilutions of NCTF $135 \mathrm{HA}^{\circledR}$ (dilutions 1/100 and $1 / 1000$ ) were added or not to the wells. After $48 \mathrm{~h}$ of culture, the supernatant was collected for collagen I determination. Collagen I was determined by spectrophotometry using Sirius red $(540 \mathrm{~nm})$. Results were expressed in $\mu \mathrm{g} /$ millions of cells.

\subsubsection{Results}

Collagen I was significantly increased by $132.2 \%$ and $114.5 \%$ in the presence of NCTF $135 \mathrm{HA}^{\circledR}$ diluted at $1 / 100$ and $1 / 1000$ compared to control group (244.9 \pm $120.1,226.2 \pm 97.6$ versus $105.5 \pm 28.6 \mu \mathrm{g} /$ millions of cells respectively; $\mathrm{p}<0.05$ ).

\subsection{Anti-Wrinkle Effect of NCTF $135 \mathrm{HA}^{\circledR}$ in GlasBox ${ }^{\text {Plus }^{\circledR}}{ }^{\text {Device by }}$ Measuring Fibroblast Contractile Forces}

\subsubsection{Objectives}

The non-cross-linked hyaluronic acid has a positive effect on the contractile force of aged fibroblasts. This study was designed to evaluate if this molecule could maintain this characteristic after combination with the polyrevitalizing complex.

\subsubsection{Materials and Methods}

\section{1) Obtaining and amplification of fibroblasts}

The study performed by Bioexigence Laboratory at Besançon, France. Normal aged fibroblasts (NF) and wrinkle fibroblasts (WF) were obtained from an operative waste (lifting) of a woman (63 years old). Classical explantation technic is used to the extraction of normal fibroblasts and wrinkle fibroblasts. Explants were cultivated in Dulbecco's modified Eagle's medium supplemented with 10\% of fetal calf serum, $40 \mathrm{mg} / \mathrm{l}$ of gentamicin and $2 \mathrm{mg} / \mathrm{l}$ of fungizone (DMEMc), in an incubator at $37^{\circ} \mathrm{C}, 5 \% \mathrm{CO}_{2}$ and $95 \%$ air. In few days, fibroblasts migrated outside the explants. Culture medium was changed twice a week and cells are subcultured by trypsinization when confluence is reached. Cells were used under 10 passages.

2) Study of contractile forces in GlasBox ${ }^{\text {Plus }}{ }^{\circledR}$ device

The GlasBox ${ }^{\text {Plus }}{ }^{\circledR}$ device is composed of a cell chamber with eight rectangular culture wells in which dermal equivalents develop. Two opposite beams hung down into each well at a distance of $27 \mathrm{~mm}$ apart (Figure 1). The dermal equivalent is attached to this sensor through a grid directly etched on the lower part of each beam. The beams have an optical system to detect their own deformation. This deformation is proportional to the contractile forces developed by dermal equivalent. The signal is collected by a computer, which include a specific program to give directly the forces in real time.

Fibroblasts are resuspended just before confluence with trypsin-EDTA (1X) solution. Fibroblasts are embedded three-dimensionally in hydrated collagen 
gels using a modified version of the technique developed by Bell et al. [12]. Briefly the mixture is composed of: 6 volumes of 1.76X (DMEMc, $\mathrm{NaHCO}_{3}$, $\mathrm{NaOH}$, antibiotics), 3 volumes rat tail type I collagen $(2 \mathrm{mg} / \mathrm{ml})$ and 1 volume of cellular suspension $\left(8 \times 10^{5} \mathrm{cells} / \mathrm{ml}\right)$. This device is a reference for measuring the function of the fibroblasts (contractile forces) since 15 years [13] [14] [15] [16]. The dermal equivalent mixture is poured into the rectangular culture plate of the GlasBox ${ }^{\text {Plus }}{ }^{\circledR}$ and polymerized in less than $30 \mathrm{~min}$ at $37^{\circ} \mathrm{C}$. Culture medium with and without NCTF $135 \mathrm{HA}^{\circledR}$ is added and the GlasBox ${ }^{\text {Plus }}{ }^{\circledR}$ is then placed into a humidified incubator at $37^{\circ} \mathrm{C}$, and forces measurements are started after 30 minutes of stabilization, for 24 hours.

Results were expressed in arbitrary unit. GlasBox ${ }^{\text {Plus }}{ }^{\circledR}$ curves were drawn and fitted to obtained maximum of contraction and area under the curve.

\subsubsection{Results}

As attempt, the contractile forces were significantly decreased in wrinkle fibroblasts compared to normal fibroblasts. Contractile forces were significantly increased with NCTF $135 \mathrm{HA}^{\circledR}$ compared to WF and significantly decreased compared to NF (Figure 2). Area under the curve and maximum of contraction were significantly increased with NCTF $135 \mathrm{HA}^{\circledR}$ compared to wrinkle fibroblasts (Figure 3).

\subsubsection{Discussion}

As the NCTF $135 \mathrm{HA}^{\circledR}$ guarantees the cells atmosphere as a protective medium without any direct effect on their biologic activities, the fibroblasts remain healthier. This product would provide the same condition as a normal cell medium which has no physiologic effect but only keep the cells in a suitable ambiance to live (Figure 4).

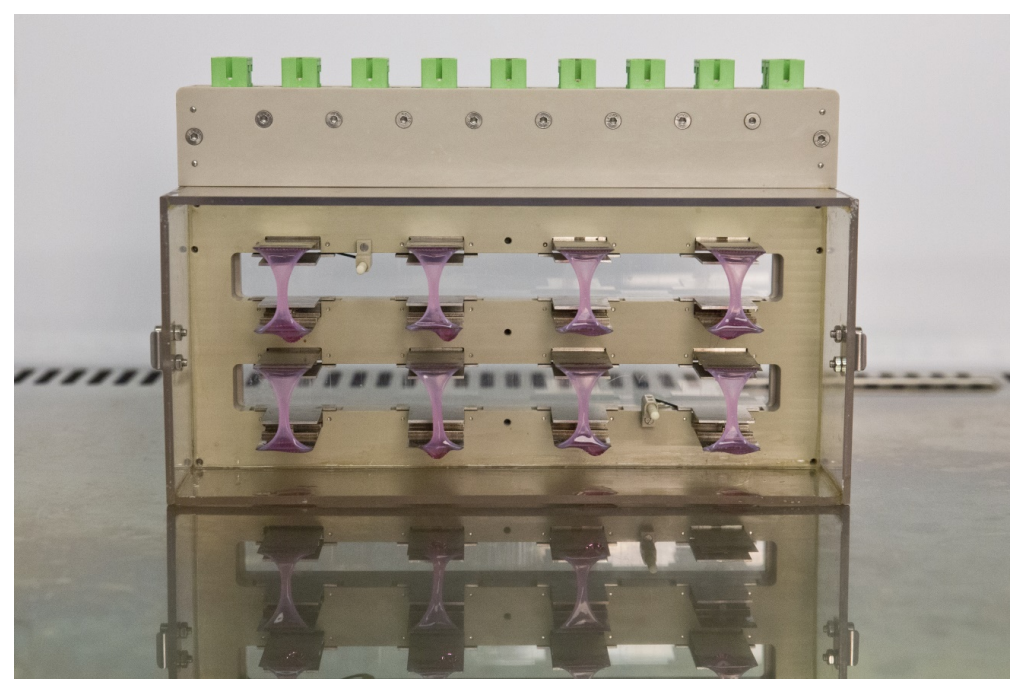

Figure 1. Equivalent dermis in GlasBox ${ }^{\text {Plus }}{ }^{\circledR}$ device. This patented device could measure the contractile forces of the human primary fibroblasts through 2 thiny electrodes in each well. The distance between the 2 plates which demonstrates the force, could be measured automatically by the device software. 


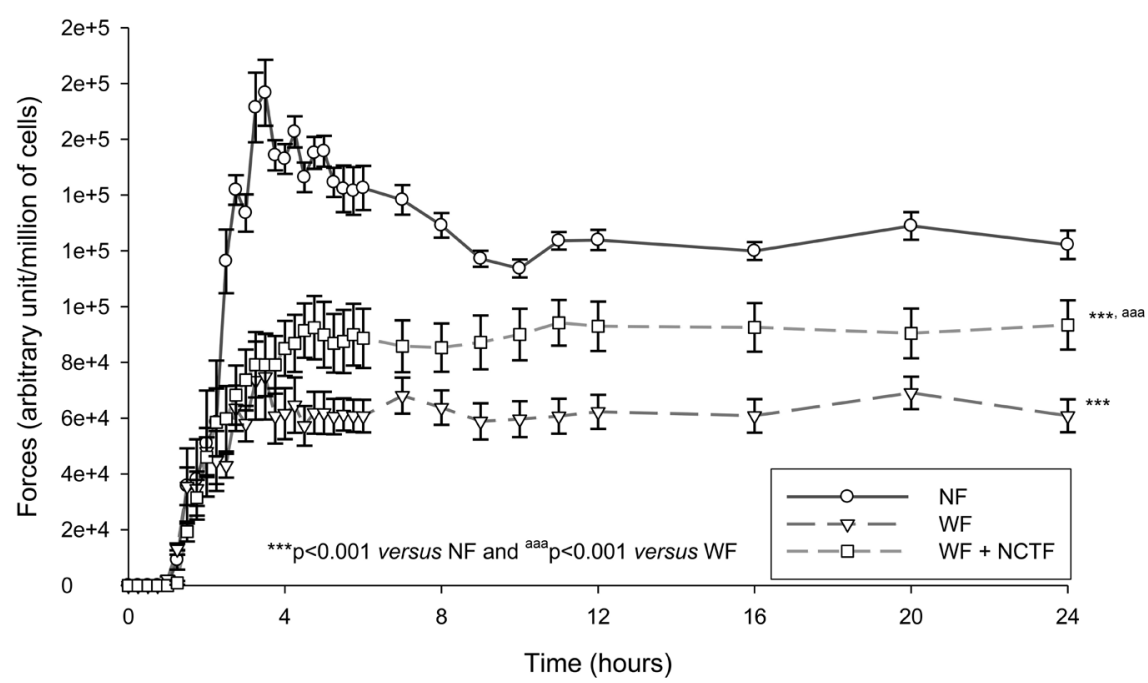

Figure 2. Contractile forces generated by NF (Normal Fibroblasts, most top curve) and WF (wrinkled fibroblasts taken from the dept of the wrinkles in the aged subjects) with NCTF (middle curve) and without NCTF (curve at the bottom). NCTF showed a significant effect on increasing the contractile forces of the WF in order to help them to show the normal fibroblasts behavior.
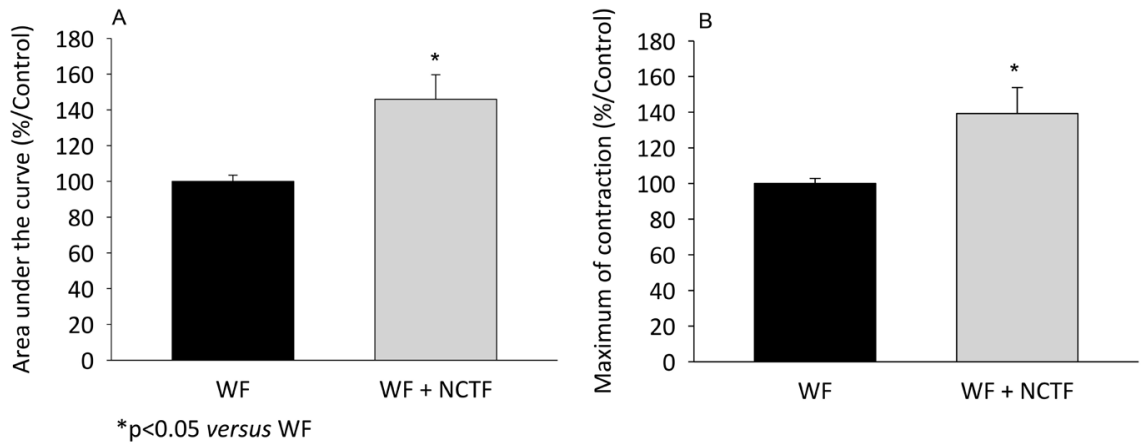

Figure 3. Area under the curve (AUC) and maximum of contraction of Glasbox curves for wrinkled fibroblasts with or without exposure to NCTF. NCTF revealed a significant increase in the AUC and also maximum contraction force of the wrinkled fibroblasts.

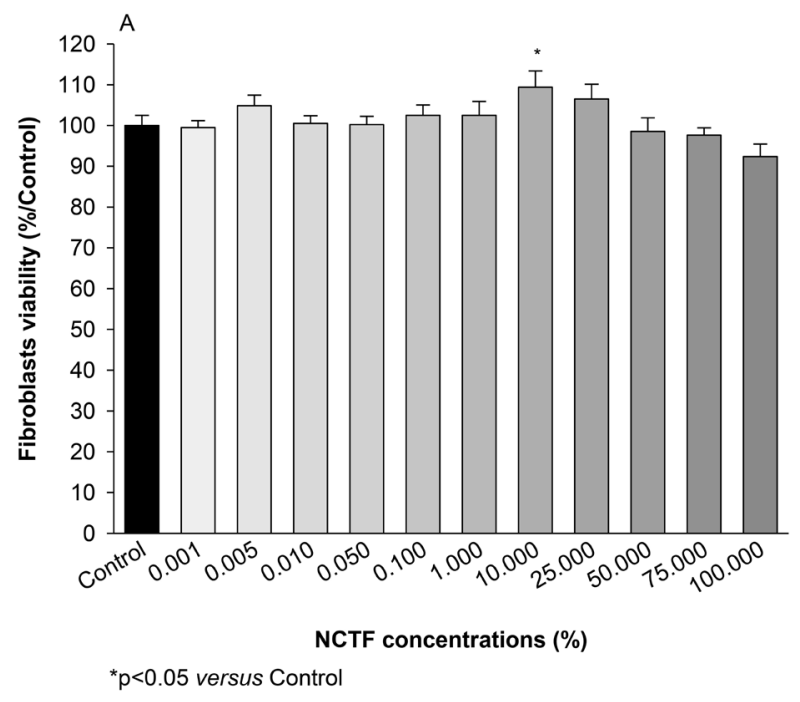




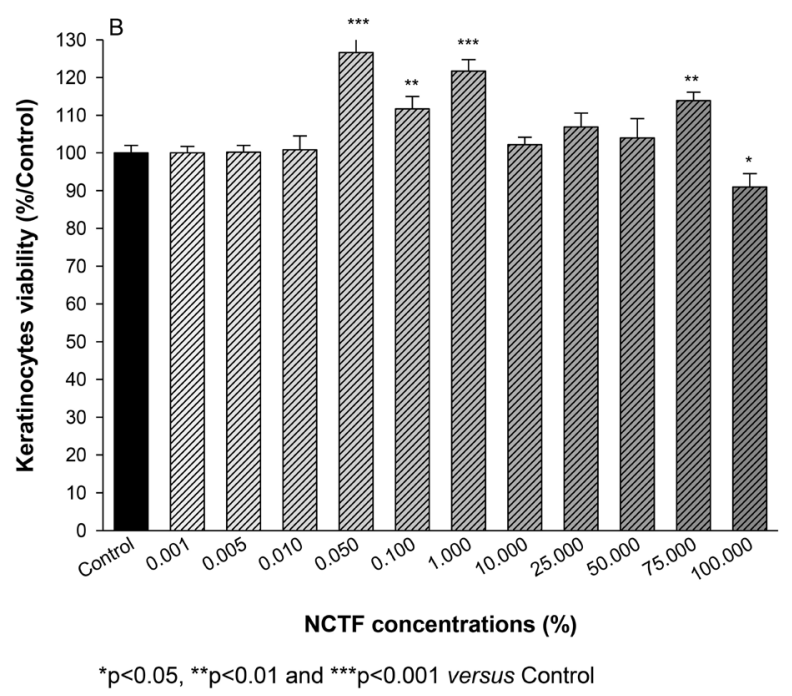

Figure 4. Viability of fibroblasts (A) and keratinocytes (B) with NCTF through MTT test. NCTF has no cytotoxic effect even with $100 \%$ concentration neither on the human primary fibroblasts nor on keratinocytes. This may show that the formulation of this product is very similar to the cell culture medium and the cells feel healthy while exposuring to this product.

\section{Ex Vivo Studies}

\subsection{Effect of NCTF $135 H^{\circledR}{ }^{\circledR}$ on GAGs Skin Content}

\subsubsection{Objectives}

The objective of this study was to evaluate if the combination of HA and polyrevitalizing component has still an effect on the glycomanioglycans content of the skin as the previous studies demonstrated that hyaluronic acid lonely increases this content [11].

\subsubsection{Materials and Methods}

Fragments of skin from 6 donors (plastic surgery) were placed in inserts of $3 \mathrm{~cm}^{2}$ positioned in culture wells with culture medium. $10 \mu \mathrm{l}$ of NCTF $135 \mathrm{HA}^{\circledR}$ were injected in the skin $\left(10 \mu \mathrm{l}\right.$ multiple points, about 10 impacts for $\left.3 \mathrm{~cm}^{2}\right)$ with $32 \mathrm{G}$ needles in comparison with control skin (untreated). Three injections were performed at days 0, 3 and 7. Photography with Life VizMicro apparatus were made before each injection for roughness analysis.

The skin fragments were fixed in formol and enclosed in paraffin after each injection for glycosaminoglycan analysis (GAGs). The GAGs of the skin (with hyaluronic acid) were stained in blue by Hale staining in histologic section. A semi-quantitative scoring of the intensity of staining in epidermis, superficial, mild and deep dermis was made (scores 0: no staining, 1: light staining, 2: moderate staining, 3: important staining and 4: very important staining).

\subsubsection{Results}

A significant decrease of roughness score was observed after 3 days of treatment compared to day $0(0.28 \pm 0.15$ versus $0.40 \pm 0.16, \mathrm{p}<0.05)$. 
No statistical significant difference was observed for GAGs content in the epidermis and superficial dermis level compared to baseline (day 0 ) after injection of NCTF $135 \mathrm{HA}^{\circledR}$. However, after 2 injections at day 0 and 3, the GAGs content seems to be increased in the deep dermis compared to Day 0 (Table 1) (Figure $5)$.

\subsubsection{Discussion}

The non-crosslinked HA existing in the formula keeps its positive effect on GAGs contents even by adding this biorevitalizing component. There is no data supporting that the presence of this component increase this positive effect versus a pure non-crosslinked HA.

\subsection{Evaluation of Filling Effect of NCTF $135 \mathrm{HA}^{\circledR}$ by Fringe Projection}

\subsubsection{Materials and Methods}

Fragments of skin from 8 donors (plastic surgery) were maintained alive at $37^{\circ} \mathrm{C}$ in phosphate buffer solution. $200 \mu$ of NCTF $135 \mathrm{HA}^{\circledR}$ or physiological serum were injected into the dermis. Acquisitions with fringe projection were performed before, just after the injection (T0), every hour during $8 \mathrm{~h}$ and after $24 \mathrm{~h}$. Topographic analyses were performed to measure the volume and the area of the papule. The ratio volume/area is an indicator of the height of the papule.

Table 1. Anti-AGE production with NCTF $135 \mathrm{HA}^{\circledR}$ on Collagen and Albumin.

\begin{tabular}{ccc}
\hline \multirow{2}{*}{ Substrates } & Anti-AGE $(\mu \mathrm{mol} / 100 \mathrm{~g})$ & Anti-AGE $(\mu \mathrm{mol} / \mathbf{1 0 0 \mathrm { g }})$ \\
& D1 & D7 \\
\hline Albumin & 60 & 1000 \\
Collagen & 410 & 820 \\
\hline
\end{tabular}

Data presenting the anti-AGE production with NCTF $135 \mathrm{HA}^{\circledR}$ after 1 and 7 days with Collagen and Albumin. AGE, Advanced Glycated End-Product; D, Day.

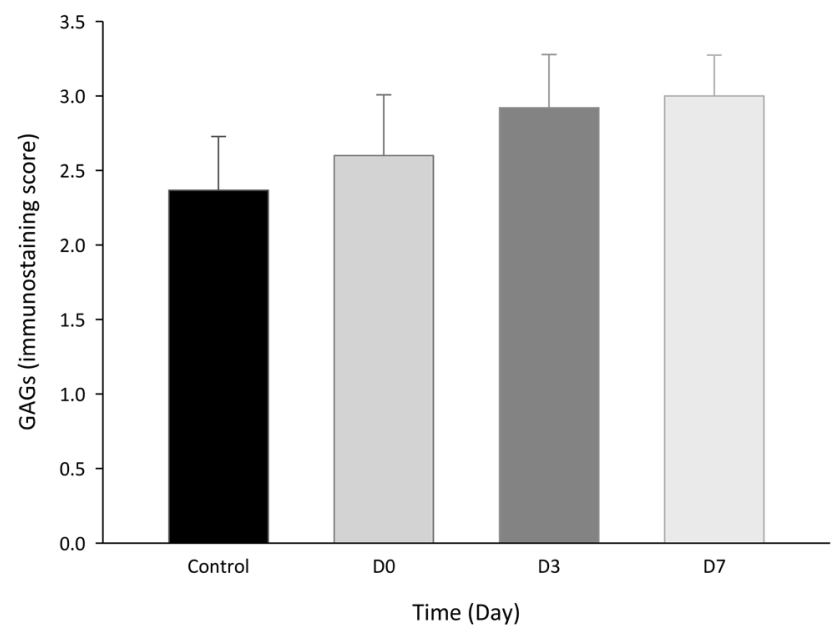

Figure 5. GAGs content in the deep dermis (measured by immunostaining score) has been increased significantly 7 days after 2 injections of NCTF at D0 and D3. 


\subsubsection{Results}

The ratio volume/area significantly decreased with time after either NCTF $135 \mathrm{HA}^{\circledR}$ or negative control (normal saline) injection. The ratio was significantly higher with NCTF $135 \mathrm{HA}^{\circledR}$ at T0 which remains higher for all study times $(1,2$, $3,4,5,6,7,8$ and 24 hour after the injection) compared to negative control ( $\mathrm{p}<$ 0.05) (Figure 6).

\subsubsection{Discussion}

Although the study product is in liquid form, it has a filling effect on the skin surface compared to the negative control (normal saline). This effect could be explained by the effect of HA in the product.

\subsection{Evaluation of the Anti-Aging Effect on Human Skin Maintained in Survival after Injection of NCTF 135HA ${ }^{\circledR}$ by Injector Gun of U225: An ex Vivo Study}

\subsubsection{Objective}

The objectives of this study were to evaluate if the injection by an automated device through different injection methods (point to point or nappage) would have any result on the collagen and elastin contents in the different levels of the skin.

\subsubsection{Materials and Methods}

Fragments of skin from 5 donors (plastic surgery) were maintained alive in culture medium at $37^{\circ} \mathrm{C}$ and $5 \% \mathrm{CO}_{2}$. Fragments were irradiated with UVA (8 $\left.\mathrm{J} / \mathrm{cm}^{2}\right)$ and UVB $\left(2 \mathrm{~J} / \mathrm{cm}^{2}\right)$ and treated or not with HA + Polyrevitalizing component. The product was injected with $\mathrm{U} 225$ meso-injector (Biophymed ${ }^{\circledR}$ ) via 2 different methods: point to point (PTP) or nappage.

After 3 days of culture, the mitotic index was evaluated by histological study (KI67). After 14 days of culture, elastin and collagen were determined with image analysis after labelling.

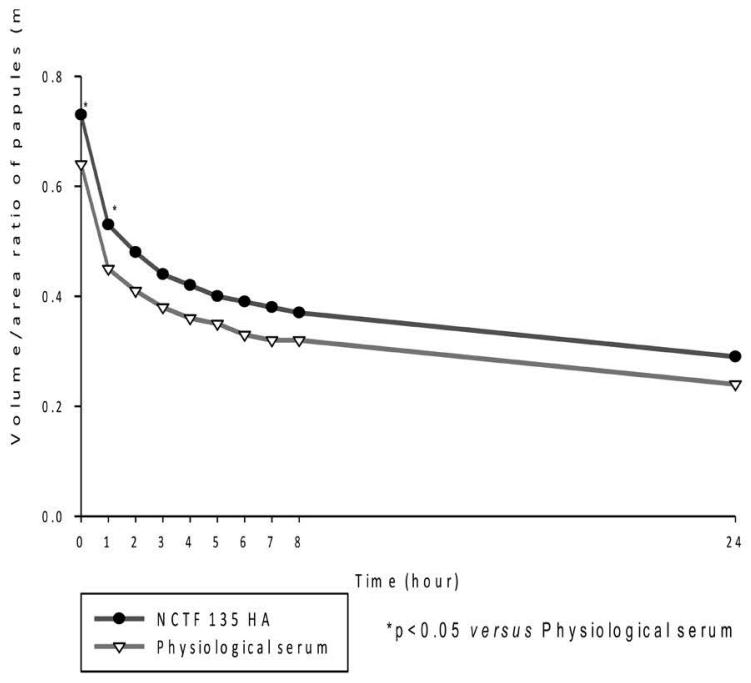

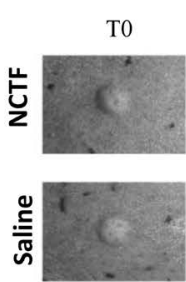

T0
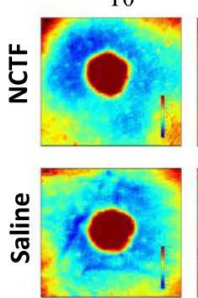
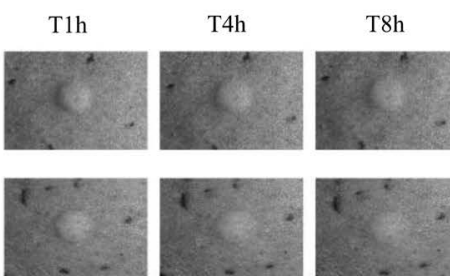

$\mathrm{T} 1 \mathrm{~h}$

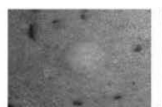

$\mathrm{T} 4 \mathrm{~h}$
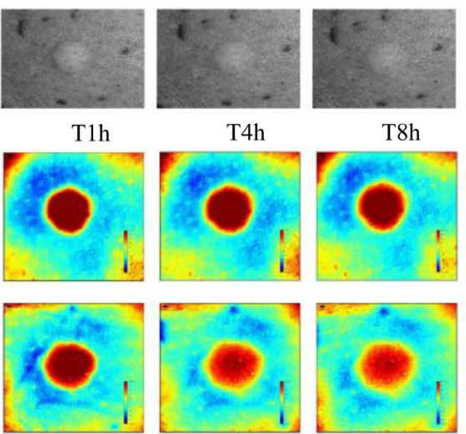

$\mathrm{T} 8 \mathrm{~h}$
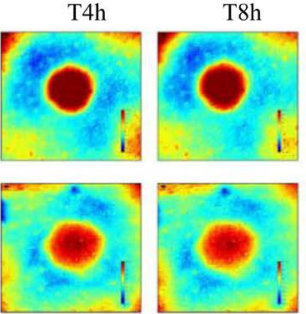

$\mathrm{T} 24 \mathrm{~h}$
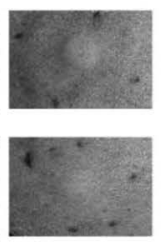

$\mathrm{T} 24 \mathrm{~h}$
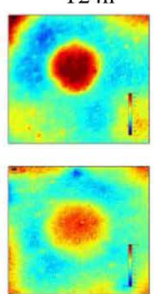

Figure 6. Evolution of the ratio volume/area of the papules measured by Fringe Projection. There is a significant difference between the volume of NCTF versus normal saline after 4 hours which remained significant till 24 hours of experience. 


\subsubsection{Results}

With the accelerating aging model induced by UV radiation, the collagen and elastin content diminished significantly versus the control skin $(\mathrm{p}=0.012$ and $\mathrm{p}$ $=0.02$ respectively). However, the elastin and collagen content were significantly increased after injection of NCTF $135 \mathrm{HA}^{\circledR}$ treatment in UV induced aged skin in different skin layers (Table 2).

\section{Clinical Trials}

\subsection{Effects of Multiple Intradermal Injections of NCTF $135 \mathrm{HA}^{\circledR}$ on Cutaneous Aging Signs: A Multicenter Non-Controlled Study}

\subsubsection{Objectives}

The primary objective of this study was to evaluate the efficacy of NCTF $135 \mathrm{HA}^{\circledR}$ on consequences of cutaneous aging on face, neck, bust line and hands, by assessing the improvement of wrinkles and fine lines, as well as for hydration, firmness and radiance of skin after 5 sessions of treatment.

The secondary objectives were to evaluate the degree of physicians and subjects satisfaction as well as the tolerance of the product.

\subsubsection{Materials and Methods}

This "open", multicenter, prospective study was conducted in 17 centers, treating 2 or 3 patients each. Recruitment was to be stopped once 40 patients had been included. Women over the age of 18 years, with no contraindications to use of NCTF $135 \mathrm{HA}^{\circledR}$, were eligible to take part in the study. Patients were to be freely selected by the practitioners. The clinical scoring performed by each investigator on a 4 scale base scoring system $(0=$ poor, $1=$ average, $2=$ good, $3=$ excellent) for wrinkles, skin hydration, skin firmness and skin radiance.

The total duration of the study was 3 months for each patient, divided into 3 phases:

- Initiation Phase: At the inclusion visit on D1, the investigator obtained the subject's written consent, once she had been given the information sheet, and the baseline skin evaluations as well as the first treatment session took place. Photographs were taken of the study zones before and then immediately after the treatment were administered.

- Repair Phase: Three treatment sessions took place on D15, D30 and D45. At each session, clinical scoring and photographs were taken of the subjects study zone before the treatment.

Table 2. Cell proliferation, elastin and collagen evaluation.

\begin{tabular}{cccc}
\hline$\%$ & Aging skin (UV) & Aging skin + HA & Statistical analysis \\
\hline Proliferation rate & $3.9 \pm 2.6$ & $8.5 \pm 2.8$ & ${ }^{*} \mathrm{p}=0.028$ \\
Elastin & $2.2 \pm 1.4$ & $4.9 \pm 2.4$ & ${ }^{*} \mathrm{p}=0.040$ \\
Collagen & $60.0 \pm 14.4$ & $75.9 \pm 7.4$ & ${ }^{*} \mathrm{p}=0.027$ \\
\hline
\end{tabular}

Data presenting the mitotic index after 3 days of culture of skin fragments irradiated or not (UVA and UVB) and with and without cocktail. Elastin and Collagen were evaluated after 14 days of culture. UV, Ultraviolet; HA, hyaluronic acid. 
- Stabilization Phase: The last treatment session took place on D75, with clinical scoring and photographs of the subjects study zones before the treatment.

- The end-of-study assessment visit took place on D90, where the last series of evaluations as well as photos was taken.

Several NCTF $135 \mathrm{HA}^{\circledR}$ administration techniques could be used, either alone or jointly, during the same treatment session such as manual multiple intradermal injection, nappage technique or injection by an injector. At the end of each treatment session, it was recommended that compression be applied to the treated area for a few minutes to prevent injection marks and that a cooling product be applied to prevent small ecchymosis.

The non-inclusion criteria were any skin disease or damage, patients with a history of autoimmune disease or receiving immunotherapy, patients with known hypersensitivity or allergy to any of the ingredients, pregnant or breastfeeding women. The investigator advised patients not to wear make-up for 12 hours following injection to avoid prolonged exposure to sunlight, UV rays or extreme cold, and not to use saunas or steam rooms for a week following injection.

\subsubsection{Outcome Measures}

Results were assessed by clinical scoring and using photographs taken with a high-quality digital camera. Primary outcome measures, scored 0 to 3 at each visit, were as follows:

- Severity of wrinkles and fine lines $(0=$ none, $1=$ not very marked, $2=$ marked, 3 = very marked);

- Skin tonicity $(0=$ poor, $1=$ average, 2 = good, $3=$ excellent $)$;

- Skin hydration $(0=$ poor, $1=$ average, $2=\operatorname{good}, 3=$ excellent $)$;

- Skin radiance $(0=$ poor, $1=$ average, $2=$ good, $3=$ excellent $)$;

- Secondary outcome measures were as follows:

- Assessment of ease of injection by the investigator ( $0=$ very satisfied, $1=$ satisfied, 2 = unsatisfied, 3 = very unsatisfied);

- Investigator's satisfaction with the product's delivery form ( $0=$ very satisfied, 1 = satisfied, 2 = unsatisfied, 3 = very unsatisfied);

- Improvement of skin appearance at the end of treatment assessed by the investigator $(0=$ no improvement, $1=$ little improvement, $2=$ improvement, 3 $=$ marked improvement, 4 = very marked improvement);

- Overall satisfaction of the investigator at the end of treatment $(0=$ very satisfied, 1 = satisfied, 2 = unsatisfied, 3 = very unsatisfied);

- Overall satisfaction of the patient at the end of treatment $(0=$ very satisfied, 1 = satisfied, 2 = unsatisfied, 3 = very unsatisfied);

- Any adverse events observed following the injections (erythema, edema, ecchymosis) were reported at each visit and rated 0 to 3 ( $0=$ none, $1=$ mild, $2=$ moderate, $3=$ severe). 


\subsubsection{Results}

\section{1) Demographic description}

40 female subjects with an average age of 51.5 years ( 29 to 80 years) were recruited by 17 practitioners with different specialties (dermatologists, aesthetic medicine specialists and plastic surgeons). These patients had obligatory the established wrinkles on the face and/or neck and/or bust-line according to the visual clinical scoring. Hands were treated only in one patient.

Subjects received all injections and underwent all control assessments scheduled by the study protocol over a period of 3 months. Two withdrawals were recorded (subjects 16 and 21) because of "lost to follow up". There were no cases of signs of local skin intolerance warranting postponement or cancellation of the next injection. Subjects skin which was otherwise healthy with wrinkles was treated in the following zones: $47 \%$ on the face only, $12 \%$ on the face, neck and bust-line, $12 \%$ on the face and neck, $10 \%$ on the face and bust-line, $10 \%$ on the bust-line only, $3 \%$ on the neck and bust-line, $3 \%$ on the neck and hands and $3 \%$ on the neck only.

2) Wrinkle score

Results of wrinkles and fine lines scoring are summarized in Table 3. The mean wrinkle score decreased significantly in repair phase (1 vs 1.8, $-46 \%, \mathrm{p}<$ $0.0001)$ and stabilizing phase $(0.9$ vs $1.8,-50 \%, \mathrm{p}<0.0001)$ but not in initial phase (Figure 7).
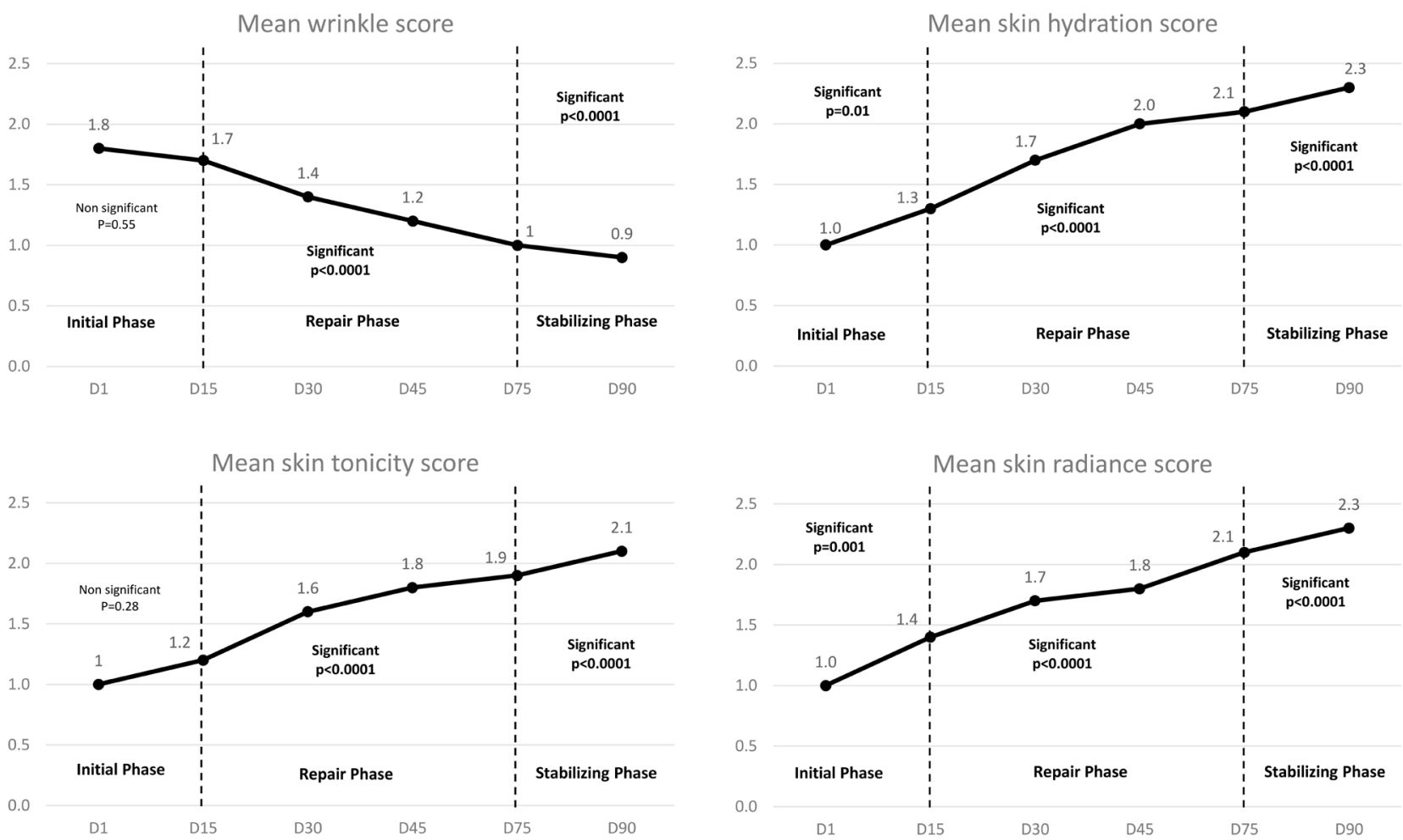

Figure 7. Evolution of mean wrinkle score, tonicity score, hydration score and radiance clinical score by time from D1 to D90 after 5 sessions of multiple intradermal injections of NCTF. The evolution is significant for all parameter as early as the third injection at D30. 
Table 3. Wrinkle clinical scoring at D1 to D90.

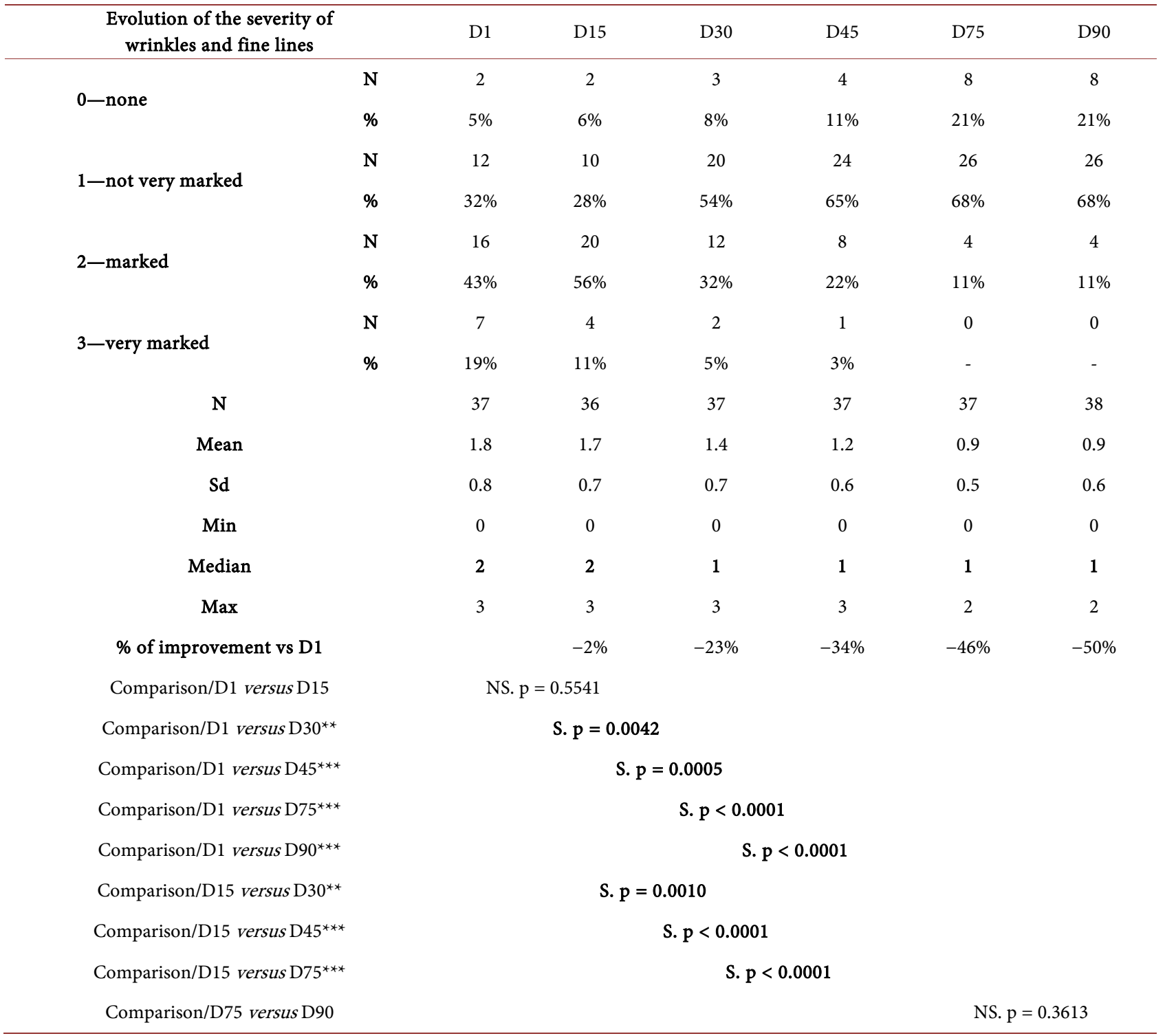

Data presenting the evolution of the severity of wrinkles and fine lines during the experimentation. N, Subject number; D, Day; sd, Standard deviation; Min, minimum; Max, Maximum; NS, not significant; S, Significant: ${ }^{* *} \mathrm{p}<0.01,{ }^{* * *} \mathrm{p}<0.001$.

\section{3) Skin Tonicity}

Mean tonicity score increased significantly in repair phase (1.9 vs $1.0,+80 \%$, p $<0.05)$ and stabilizing phase $(2.1$ vs $1.0,110 \%, \mathrm{p}<0.05)$ but not in initial phase (Figure 7).

4) Skin Hydration clinical scoring

Mean hydration score increased significantly in all phases: initial phase by $30 \%$ ( 1.3 vs $1.0, \mathrm{p}<0.05)$, repair phase by $110 \%(2.1$ vs $1.0, \mathrm{p}<0.05)$ and stabilizing phase by $130 \%$ (2.3 vs 1.0, p < 0.05) (Figure 7).

5) Skin Radiance clinical scoring

Mean radiance score increased significantly in all phases: initial phase by $40 \%$ (1.4 vs $1.0, \mathrm{p}<0.05)$, repair phase by $110 \%(2.1$ vs $1.0, \mathrm{p}<0.05)$ and stabilizing 
phase by $130 \%(2.3$ vs $1.0, \mathrm{p}<0.05)$ (Figure 7).

6) Subjects satisfaction score

A visible skin improvement was observed by $97 \%$ of subjects: $18 \%$ Very marked improvement, 37\% Marked improvement, 42\% Improvement, 3\% Slight improvement and $0 \%$ No improvement.

The satisfaction rate by subjects reported 97\%: 55\% very satisfied, $42 \%$ satisfied and only $3 \%$ of subjects were unsatisfied.

\subsection{Evaluation of the Anti-Aging Effect of NCTF $135 \mathrm{HA}^{\circledR}$ on Healthy Subjects through Instrumental Measures: A Monocentric Non-Controlled Study}

\subsubsection{Objectives}

The primary objective of this study was to evaluate the efficacy of NCTF $135 \mathrm{HA}^{\circledR}$ on cutaneous aging on the face, neck and bust-line, by assessing the improvement obtained for wrinkles, as well as for skin pore size, thickness and density of the dermis and homogeneity of complexion.

The secondary objective was to evaluate the degree of satisfaction of investigator and subjects as well as the tolerance of the product.

\subsubsection{Materials and Methods}

20 women (between 35 and 65 years old) were enrolled for this study.

The total schedule duration of the study was 4 months for each patient with:

- Inclusion visit at D0, the investigator obtained the subject's written consent, once she had been given the information sheet;

- Skin evaluation at D0 (before injection), D15, D30, D60, D90 and D120;

- Injection treatment at D0, D15, D30, D60 and D90;

The evaluation criteria used were:

- Macrophotography with Proscope ${ }^{\oplus}$ for the measurement of pore size in $\mu \mathrm{m}$,

- Cutaneous High Frequency Ultrasound Imageing (HFUI) to evaluate the density and the thickness of dermis;

- Chromametric analysis with chromameter Konica Minolta CR/DP- $400^{\circledR}$ to evaluate the skin homogeneity after the treatment. Wrinkle depth by clinical scoring (scores 0 to 4,4 corresponding to very deep wrinkle);

- Skin radiance by clinical scoring (scores 0 to 4,4 corresponding to dull skin).

\subsubsection{Results}

1) Demographic description

20 women aged $49.3 \pm 9.3$ years were enrolled in this study. A good tolerance for the product was observed. Two women came out of the study, the first for medical reason with no relation with the treatment and the second for allergy.

2) Pores size

Pores size was significantly decreased as early as the second injection (D15, $\mathrm{p}$ $<0.05$ ) and was continued to the end of the experiment at D30, D60, D90 and D120 ( $<<0.001$, Figure 8). 

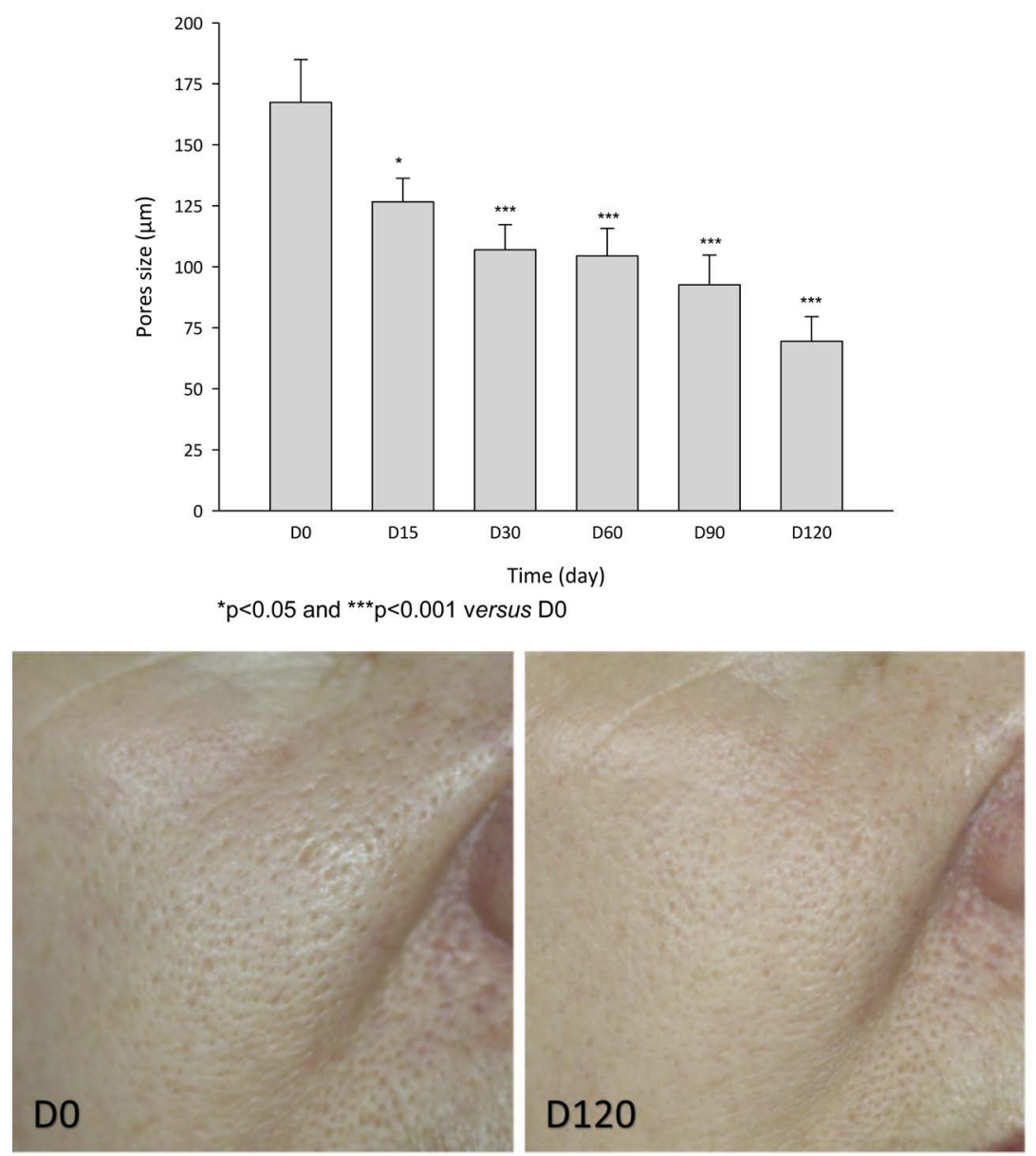

Figure 8. Pores size measured by Proscope ${ }^{\circledast}$ was decreased significantly after intradermal injection of NCTF as early as D15, it means after only one injection sessions. This significant decrease continued progressively till D120 (4 months after the first inhjection).

3) Density and thickness of the dermis

The density and the thickness of the dermis were significantly increased as early as D30 ( $3^{\text {rd }}$ injection session, $\left.\mathrm{p}<0.01\right)$ and was continued to be improved atD60, D90 and D120 compared to D0 ( $<<0.001$, Figure 9).

4) Skin homogeneity

The skin homogeneity was measured by the difference of the L parameter (in $\mathrm{L}, \mathrm{a}^{*}, \mathrm{~b}^{\star}$ colorimetric system) between the internal and external area of the cheek. Skin homogeneity was significantly increased (delta of 2 points decreased) as early as the second injection (D15, p < 0.01) to the end of the study at D30 ( $<<0.01)$, D60 and D90 ( $<<0.05)$ and D120 $(\mathrm{p}<0.001)$ (Figure 10).

$5)$ Wrinkle depth and volume

Wrinkle depth measured by profilometric device (Skinstation ${ }^{\oplus}$ ) was significantly decreased at D90 and D120 compared to D0 ( $\mathrm{p}<0.05)$. In total, the wrinkle depth was decreased by $43.32 \%$ after 5 mesotherapy treatments. By the same device, the volume of the wrinkles was significantly decreased at D60 $(\mathrm{p}<0.05)$, D 90 and D120 compared to D0 ( $<0.01$ ) after NCTF 135HA ${ }^{\circledR}$ injection (Figure 11). 


\section{6) Skin radiance}

The score of skin radiance was significantly increased as early as 15 days of treatment which was continued to increase until the end of the experiment compared to D0 ( $\mathrm{p}<0.001$ for all study visits). In total, the skin radiance was increased by $49.3 \%$ after 5 mesotherapy treatments at D120) (Figure 12).
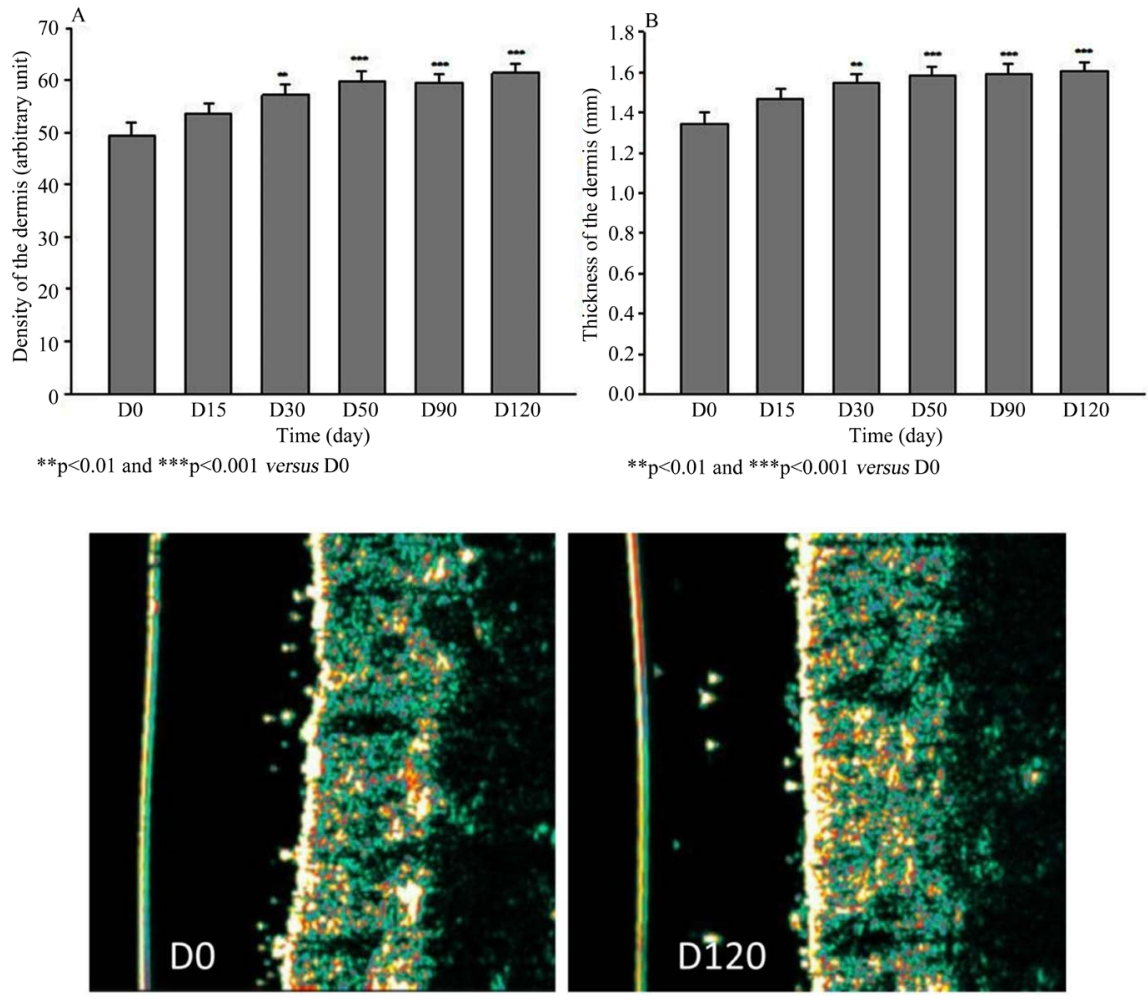

Figure 9. Density and thickness of the dermis has been increased significantly as early as day 30 (after 2 injection sessions) which continued to increase till day 120 .

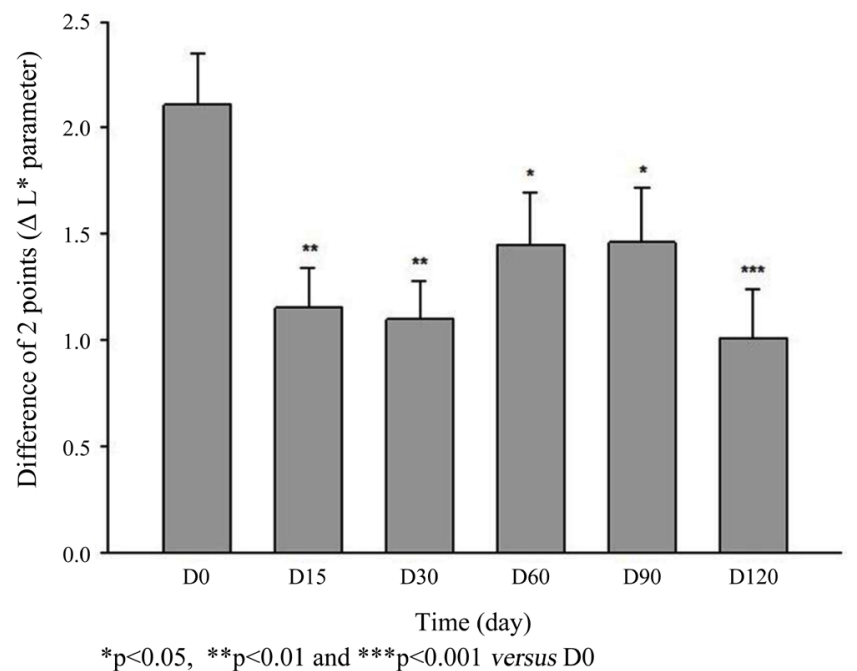

Figure 10. Skin homogeneity was increased over time as the difference of $L^{\star}$ parameter $\left(\mathrm{L}^{*} \mathrm{a}^{*} \mathrm{~b}^{*}\right.$ system) between two points on the external and internal part of the cheek was diminished significantly as early as day 15 it means even after 1 injection session. 

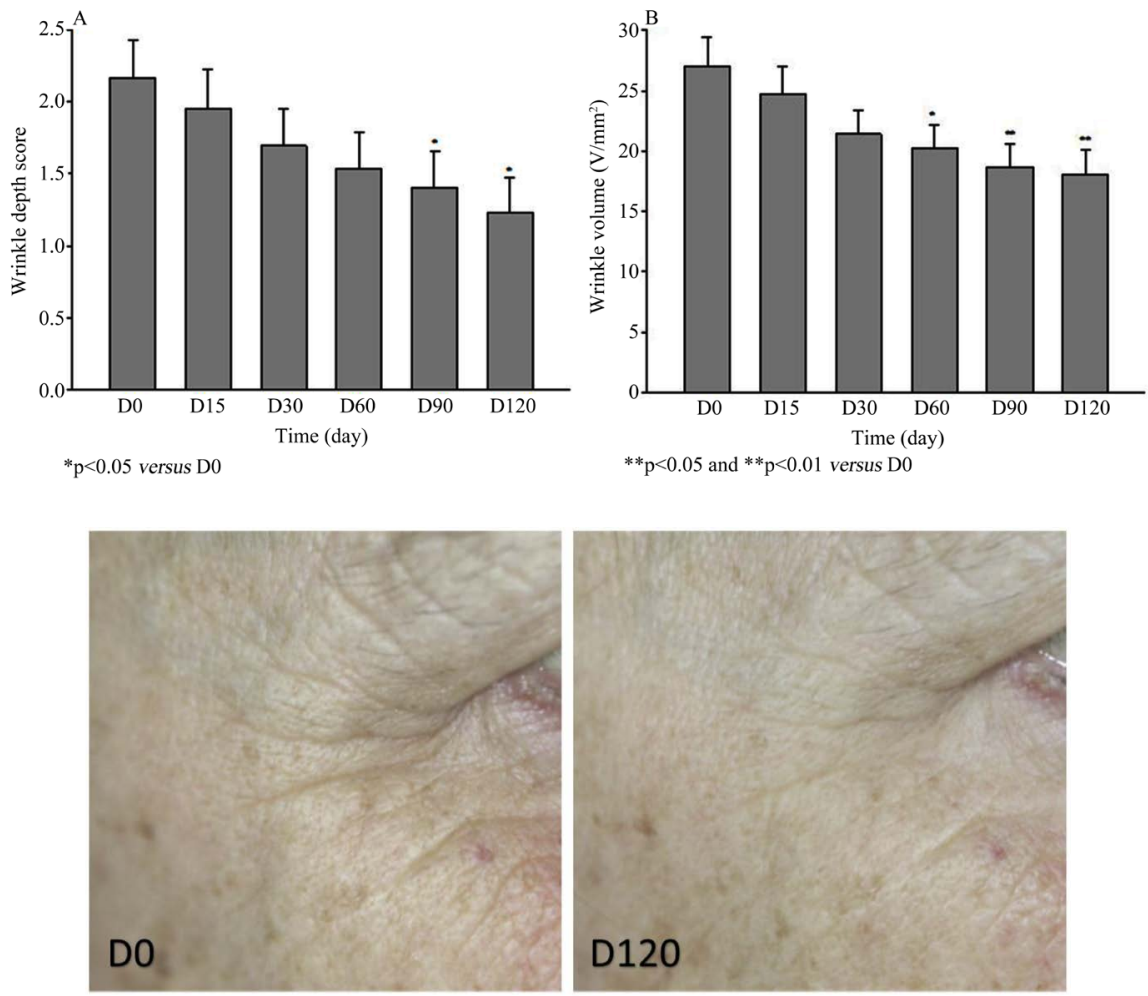

Figure 11. Wrinkle depth score and wrinkle volume measured by SkinStation ${ }^{\circledR}$ was diminished significantly at D90 (3 months) and D60 (2 months) respectively.

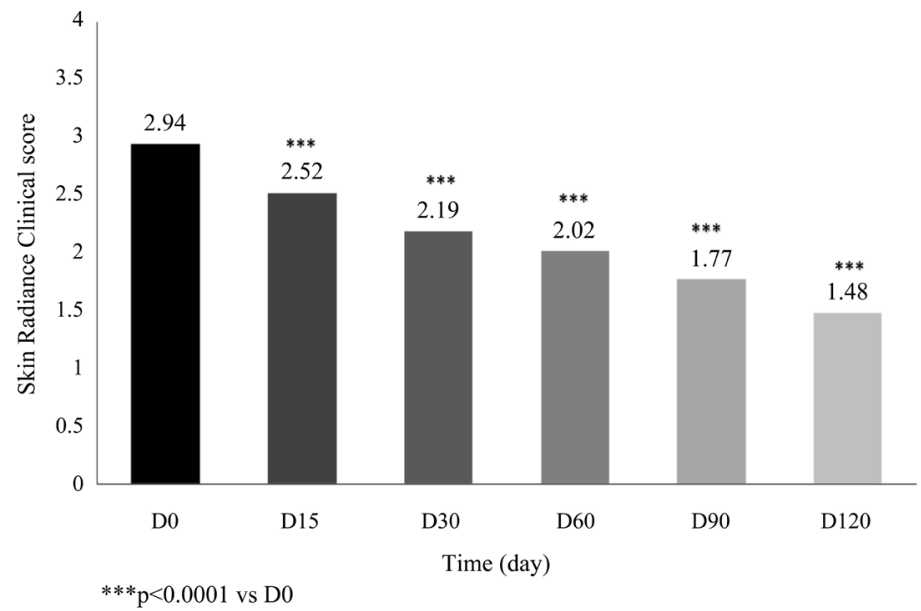

Figure 12. Skin radiance measured by Skin Dullness Clinical Scoring ( 0 for very radiant skin and 4 for very dull skin). The skin dullness was diminished significantly as early as day $15(\mathrm{p}<0.0001)$ it means following only one injection.

\section{Discussion}

The aim of this study was to show the anti-aging effect of NCTF $135 \mathrm{HA}^{\circledR}$ intradermal multi-injections. Skin aging can be due to intrinsic, natural and genetically determined factors, as well as extrinsic lifestyle-driven and environmental factors [1] [10]. It is characterized by a number of objective physical indicators, including skin dryness, laxity and poor elasticity, color and surface irregularity, 
formation of pronounced skin markings, and wrinkles of different intensity. Fibroblasts represent the major role in producing and maintaining the extracellular connective tissue that is crucial to maintaining the youthful appearance of the skin [17].

The first part of the study was dedicated to the in vitro effect of NCTF $135 \mathrm{HA}^{\circledR}$. This part demonstrated that NCTF $135 \mathrm{HA}^{\circledR}$ increase fibroblasts collagen I expression and has a good anti-glycation effect thanks to the hyaluronic acid content. Jäger et al. have demonstrated that NCTF $135 \mathrm{HA}^{\circledR}$ maintain cell proliferation and induce enhance mRNA expression of collagen I, MMP-1 (Matrix Metallo-Proteinase) and TIMP-1 (Tissue Inhibitor of Matrix Metallo-Proteinase) in fibroblasts for at least 11 days in culture [18]. They hypothesize that the induction of collagen I degradation by MMP-1 could be counteracted by the concomitant expression of TIMP-1 allowing sustained collagen I production. This argument has been already supported by previous studies suggesting that hyaluronic acid can induce collagen I in fibroblasts [19]. It could thus be speculated that sensitization of fibroblasts by hyaluronic acid is elicited via interaction through various cellular receptors including CD44 [20].

Wrinkle fibroblasts generated less mechanical forces compared to non-wrinkle fibroblasts of the same donors [21]. Skin is physiologically tensed. During aging, strain forces inside the skin decreases and subsequent relaxation are responsible for wrinkles formation. The in vitro study by GlasBox ${ }^{\text {Plus }}{ }^{\circledR}$ demonstrated that the addition of NCTF $135 \mathrm{HA}^{\circledR}$ increase contractile forces generated by wrinkle fibroblasts thanks to the HA component and has probably an anti-wrinkle effect acting on collagen. It is becoming increasingly evident that the optimal function of fibroblasts is critically dependent on their reciprocal mechanical interactions with their surrounding microenvironment [22]. Mechanical tension between fibroblasts and the surrounding extracellular matrix appears to be critical for fibroblast function and normal balanced production of collagen and collagen-degrading enzymes.

Both in vivo studies have shown main advantages of biorevitalizing solutions for skin rejuvenation. Injections were easy to perform with low pain and limited side effects (one subject only was excluded in the first clinical trial on 2011). Clinical evaluation showed a decrease in wrinkle depth after NCTF $135 \mathrm{HA}^{\circledR}$ injections. In the same time, the density and the thickness of dermis were significantly increased after several injections. An upgrade in skin tonicity, hydration and radiance were observed.

\section{Conclusions}

Microfillers are the non-cross-linked hyaluronic acid fillers which are boosted by a complex of nutrients such as vitamins, antioxydants, aminoacids, etc. in order to protect the HA component. These microfillers, previously named as "biorevitalizing solutions" have a remarkable accumulating role in preventing the signs of skin aging. 
In the past few years, there are many new products in this field but the authors summarized all studies performed during last 10 years on one the pioneer product named NCTF $135 \mathrm{HA}^{\circledR}$ as a good representative for this category of products.

In Vitro, ex vivo and in vivo studies showed that this minimally invasive technique could be used in order to improve the skin quality which could have a visual effect on preventing the aging signs. The long term cohorts need to prove this hypothesis.

\section{Disclosure}

The authors report no conflicts of interest in this work. FF is the Scientific Director and employee of FILLMED Laboratories.

\section{Conflicts of Interest}

The authors declare no conflicts of interest regarding the publication of this paper.

\section{References}

[1] Farage, M.A., Miller, K.W., Elsner, P. and Maibach, H.I. (2013) Characteristics of the Aging Skin. Advances in Wound Care (New Rochelle), 2, 5-10. https://doi.org/10.1089/wound.2011.0356

[2] Waller, J.M. and Maibach, H.I. (2005) Age and Skin Structure and Function, a Quantitative Approach (I): Blood Flow, pH, Thickness, and Ultrasound Echogenicity. Skin Research and Technology, 11, 221-235. https://doi.org/10.1111/j.0909-725X.2005.00151.x

[3] Boss, G.R. and Seegmiller, J.E. (1981) Age-Related Physiological Changes and Their Clinical Significance. The Western Journal of Medicine, 135, 434-440.

[4] McCallion, R. and Li Wan Po, A. (1993) Dry and Photo-Aged Skin: Manifestations and Management. Journal of Clinical Pharmacy and Therapeutics, 18, 15-32. https://doi.org/10.1111/j.1365-2710.1993.tb00562.x

[5] Südel, K.M., Venzke K., Mielke, H., Breitenbach, U., Mundt, C., Jaspers, S., Koop, U., Sauermann, K., Knussman-Hartig, E., Moll, I., Gercken, G., Young, A.R., Stäb, F., Wenck, H. and Gallinat, S. (2005) Novel Aspects of Intrinsic and Extrinsic Aging of Human Skin: Beneficial Effects of Soy Extract. Photochemistry and Photobiology, 81, 581-587. https://doi.org/10.1562/2004-06-16-RA-202.1

[6] Duncan, K.O. and Leffell, D.J. (1997) Preoperative Assessment of the Elderly Patient. Dermatolic Clinics, 15, 583-593. https://doi.org/10.1016/S0733-8635(05)70468-X

[7] Haneke (2006) Skin Rejuvenation without a Scalpel. I. Fillers. Journal of Cosmetic Dermatology, 5, 157-167. https://doi.org/10.1111/j.1473-2165.2006.00243.x

[8] Savoia, A., Landi, S. and Baldi, A. (2013) A New Minimally Invasive Mesotherapy Technique for Facial Rejuvenation. Dermatology and Therapy, 8, 83-93. https://doi.org/10.1007/s13555-012-0018-2

[9] Lorizzo, M., De Padova, M.P. and Tosti, A. (2008) Biorejuvenation: Theory and Practice. Clinics in Dermatology, 26, 177-181. https://doi.org/10.1016/j.clindermatol.2007.09.011

[10] Prikhnenko, S. (2015) Polycomponent Mesotherapy Formulations for the Treatment of Skin Aging and Improvement of Skin Quality. Clinical, Cosmetic and In- 
vestigational Dermatology, 8, 151-157. https://doi.org/10.2147/CCID.S76721

[11] Zhao, J.Y., Chai, J.K., Song, H.F. and Xu, M.H. (2012) Experimental Study of Hyaluronic Acid on the Biomechanical Compliance of Porcine Acellular Dermal Transplantation. The National Medical Journal of China, 92, 1428-1430.

[12] Bell, E., Ivarsson, B. and Merril, C. (1979) Production of a Tissue-Like Structure by Contraction of Collagen Lattices by Human Fibroblasts of Different Proliferative Potential in Vitro. Proceedings of the National Academy of Sciences of the United States of America, 3, 1274-1278. https://doi.org/10.1073/pnas.76.3.1274

[13] Armbruster, V., Gharbi, T., Viennet, C. and Humbert, Ph. (2004) Silicon Grid Devices for Attachment of Cultured Fibroblast Collagen Lattices. Sensor and Actuators A: Physical, 116, 219-223. https://doi.org/10.1016/j.sna.2004.03.065

[14] Robin, S., Courderot-Masuyer, C., Tauzin, H., Guillon, S, Gaborit, J., Harbon, S. and Humbert, Ph. (2012) Evaluation of Protective and Restoring Effects of a Mixture of Silanols on Photoaging. Use of a Device Allowing the Quantification of Contractile Strengths of Human Fibroblasts after UVA Irradiation. International Journal of Cosmetic Science, 34, 311-317. https://doi.org/10.1111/j.1468-2494.2012.00716.x

[15] Humbert, Ph., Fanian, F., Lihoreau, T., Jeudy, A., Elkhyat, A., Robin, S., CourderotMasuyer, C., Tauzin, H., Lafforgue, C. and Haftek, M. (2015) Mecano-Stimulation of the Skin Improves Sagging Score and Induces Beneficial Functional Modification of the Fibroblasts: Clinical, Biological and Histological Evaluations. Clinical Interventions in Aging, 10, 387-403. https://doi.org/10.2147/CIA.S69752

[16] Courderot-Masuyer, C., Robin, S., Tauzin, H. and Humbert, Ph. (2016) Evaluation of Lifting and Antiwrinkle Effects of Calcium Hydroxylapatite Filler. In Vitro Quantification of Contractile Forces of Human Wrinkle and Normal Aged Fibroblasts Treated with Calcium Hydroxylapatite. Journal of Cosmetic Dermatology, 15, 260-268. https://doi.org/10.1111/jocd.12215

[17] Varani, J. (2010) Fibroblast Aging: Intrinsic and Extrinsic Factors. Drug Discovery Today: Therapeutic Strategies, 7, 65-70. https://doi.org/10.1016/j.ddstr.2011.06.001

[18] Jäger, C., Brenner, C., Habicht, J. and Wallich, R. (2012) Bioactive Reagents Used in Mesotherapy for Skin Rejuvenation in Vivo Induce Diverse Physiological Processes in Human Skin Fibroblasts in Vitro-A Pilot Study. Experimental Dermatology, 21, 72-75. https://doi.org/10.1111/j.1600-0625.2011.01400.x

[19] Wang, F., Garza, L.A., Kang, S., Varani, J., Orringer, J.S., Fisher, G.J. and Voorhees, J.J. (2007) In Vivo Stimulation of de Novo Collagen Production Caused by Cross-Linked Hyaluronic Acid Dermal Filler Injections in Photodamaged Human Skin. Archives of Dermatology, 143, 155-163.

https://doi.org/10.1001/archderm.143.2.155

[20] Weindl, G., Schaller, M., Schäfer-Korting, M. and Korting, H.C. (2004) Hyaluronic Acid in the Treatment and Prevention of Skin Diseases: Molecular Biological, Pharmaceutical and Clinical Aspects. Skin Pharmacology and Physiology, 17, 207-213. https://doi.org/10.1159/000080213

[21] Courderot-Masuyer, C. (2017) Mechanical Properties of Fibroblasts. In: Humbert, P., Fanian, F., Maibach, H. and Agache, P., Eds., Agache's Measuring the Skin, Springer, Cham, 903-909. https://doi.org/10.1007/978-3-319-32383-1_125

[22] Vedrenne, N., Coulomb, B., Danigo, A., Bonté, F. and Desmoulière, A. (2012) The Complex Dialogue Between (Myo)fibroblasts and the Extracellular Matrix during Skin Repair Processes and Ageing. Pathologie Biologie (Paris), 60, 20-27. https://doi.org/10.1016/j.patbio.2011.10.002 